\title{
Continuous network structure of two-dimensional silica across a supporting metal step edge: An atomic scale study
}

\author{
Leonard Gura $\odot,{ }^{1}$ Sergio Tosoni $\odot,{ }^{2}$ Adrián Leandro Lewandowski $\odot,{ }^{1}$ Patrik Marschalik, ${ }^{1}$ Zechao Yang $\odot,{ }^{1}$ \\ Wolf-Dieter Schneider (1), ${ }^{1}$ Markus Heyde $(1),{ }^{1, *}$ Gianfranco Pacchioni $\odot{ }^{2},{ }^{2}$ and Hans-Joachim Freund (1) ${ }^{1}$ \\ ${ }^{1}$ Fritz-Haber-Institut der Max-Planck-Gesellschaft, Faradayweg 4-6, 14195 Berlin, Germany \\ ${ }_{2}^{2}$ Dipartimento di Scienza dei Materiali, Università di Milano-Bicocca, Via R. Cozzi, 55, Milan, Italy
}

(Received 12 November 2020; revised 2 March 2021; accepted 29 June 2021; published 29 July 2021)

\begin{abstract}
The network structure of a silica bilayer film at a monolayer-bilayer transition and across a supporting metal step edge was studied at the atomic scale by scanning tunneling microscopy. The ring size distribution, ring-ring distances, and height profiles are analyzed across the step edge region. Density functional theory proposes two models to explain the observed network structure: a pinning of the lower layer to the substrate and a carpetlike mode. The results indicate a continuous coverage of the silica bilayer film across the step edge.
\end{abstract}

DOI: 10.1103/PhysRevMaterials.5.L071001

Thin oxide films are of high technological importance for electronics and catalysis [1,2]. So far the only known large area two-dimensional (2D) oxide that is liftable from its substrate is 2D silica [3-5]. Due to its chemical inertness, its insulating properties, and its structural diversity the film system is promising for various applications, amongst them 2D heterostructures [6-8]. These applications are often limited by anisotropic properties and the lattice mismatch. The glassforming capability of the silica network can help to overcome these constraints. For application, the integrity of the vitreous film against reactive conditions, such as reactive metal step edges at elevated temperatures, must be verified. Therefore, we need to investigate its atomic structure at the mesoscopic scale.

Various 2D silica polymorphs have been reported [9]. The silica monolayer (ML) is chemically coupled to the metal substrate, while the silica bilayer (BL) exhibits a weak coupling to the metal substrate due to van der Waals bonding. 2D silica bilayer films can be crystalline or vitreous depending on the preparation conditions. The different phases, namely, the monolayer, zigzag, and bilayer phase can coexist. Such a coexistence, as reported in Ref. [10], causes a stepped topography at the ML-BL transition. A stepped topography can also be observed for underlying substrate step edges. The open question is, whether the films are connected across this stepped topography. To answer this question is important because for most technological applications like 2D heterostructures the ability to form continuous coverages is

*heyde@ fhi-berlin.mpg.de

Published by the American Physical Society under the terms of the Creative Commons Attribution 4.0 International license. Further distribution of this work must maintain attribution to the author(s) and the published article's title, journal citation, and DOI. Open access publication funded by the Max Planck Society. crucial. In this study, we elucidate the atomic structure of a vitreous silica bilayer in the vicinity of a ML-BL transition and of a BL with an underlying substrate step edge.

In literature, several other film systems have been investigated across substrate steps. For some of the film systems with a weak coupling to the substrate, a carpet mode was reported [11-18]. Also crystalline oxides with strong covalent and ionic bonds can cover substrate step edges continuously $[19,20]$. For the graphene synthesis, metal step edges play an important role [21-23]. The continuous growth of graphene over substrate step edges was already observed by scanning tunneling microscopy (STM) [24-26]. Graphene can show a continuous growth mode with a pinning to the substrate [27]. To our knowledge, so far only crystalline thin-film systems have been studied on substrate step edges. We present the continuous coverage of a vitreous silica bilayer system across a $\mathrm{Ru}(0001)$ step edge. The experimental findings are supported by density functional theory (DFT) calculations. To account for computational limits, an optimized system of a crystalline silica bilayer film on a stepped $\mathrm{Ru}$ surface has been modeled. We elaborate on models of a pinned film and a carpetlike mode.

In previous publications, the structural properties of the metal supported silica bilayer were described thoroughly $[28,29]$. The internal structure of the bilayer film system has been verified. The upper and lower layers are connected over straight Si-O-Si bonds [5]. Exfoliation experiments, which revealed the flexibility and mechanical stability of the silica bilayer, proved that the film is freely transferable [3,4]. Reaction fronts were observed in the confined space between film and substrate with low-energy electron microscopy [30]. The film transfer and the propagation of the reaction fronts affirm a continuous and smooth coverage - also across substrate step edges.

In the present investigation, we use a nonaveraging technique to investigate the structural and mesoscopic features of a vitreous bilayer film at a ML-BL transition and a $\mathrm{BL}$ 
across a metal step edge. Due to the lack of periodicity and order, vitreous films do not have a registry with the underlying substrate. Therefore, the film on the upper and lower substrate terraces cannot be related to prove the continuity of the film. A detailed analysis of STM data has been performed. We resolved the ring structure of the vitreous silica bilayer at the ML-BL transition and across the $\mathrm{Ru}(0001)$ step edge. Based on the detected network, we evaluate the ring size distribution, characteristic ring, and atomic distances. DFT calculations were performed to study possible scenarios for a continuous coverage of the silica BL across the $\mathrm{Ru}(0001)$ step edge. The topographies and characteristic distances from the theoretical models are compared to those deduced from STM measurements.

We applied room-temperature and low-temperature STMs with PtIr tips, described in Refs. [31,32]. The microscopes operate in ultrahigh vacuum (UHV) at a base pressure range of $10^{-10}$ mbar. Prior to film growth, the $\mathrm{Ru}(0001)$ single crystal was repeatedly cleaned by $\mathrm{Ar}^{+}$bombardment, annealing in UHV at $1450-1500 \mathrm{~K}$ for $1 \mathrm{~min}$, and annealing in oxygen atmosphere of $2 \times 10^{-6}$ mbar at $1250 \mathrm{~K}$ for $20 \mathrm{~min}$. The surface quality was checked by STM and lowenergy electron diffraction measurements. The silica film preparation starts with a $3 \mathrm{O}-(2 \times 2)$-precoverage, followed by an annealing in $2 \times 10^{-6}$ mbar oxygen atmosphere at $1150-1200 \mathrm{~K}$. Subsequently, silicon is deposited by physical vapor deposition at an oxygen pressure of $2 \times 10^{-7}$ mbar. Finally, the film is annealed in $2 \times 10^{-6}$ mbar oxygen pressure at $1150-1200 \mathrm{~K}$. The result is an atomically flat silica film with vitreous domains. DFT calculations were performed with the GPU-accelerated version of the code VASP 6 [33-36]. The projector-augmented-wave pseudopotentials $[37,38]$ were adopted and the basis set was expanded within a kinetic cutoff of $400 \mathrm{eV}$. We adopted the Perdew, Burke and Ernzerhof [39] functional corrected for the long-range dispersion with the semiempirical DFT + D2' approach [40,41].

Our STM measurements reveal the coexisting silica ML and $\mathrm{BL}$ film on $\mathrm{Ru}(0001)$. Figure 1 shows the transition of the two phases, which were analyzed individually in Ref. [10]. The here presented network is based on a semiautomated network detection program, which is outlined in Sec. 1 of the Supplemental Material [42]. Figure 1(b) reveals that the program detects the crystalline network with a domain boundary in the ML and the vitreous network in the BL phase. This is in agreement with Ref. [10]. However, the detected network differs at the ML-BL transition. The ring-ring distances are increased compared to the uniform ring-ring distance distribution in the ML and BL, respectively. Furthermore, the ring connectivity is not reliable at the transition region. This affects also the ring sizes of nearest neighbor rings in the ML and BL films. As shown in Fig. 1(a), the image intensity is increased at the rim of the ML-BL transition. This characteristic image contrast at the rim can be caused by dangling bonds or chemically bound molecules. This indicates that the upper layer of the vitreous bilayer is not connected to the ML phase. Hence, for the ML-BL transition a fully connected film could not be detected.

While the ML-BL transition in Fig. 1 resembles a stepped topography inside the film system, an underlying substrate step imposes a stepped topography upon the film system.
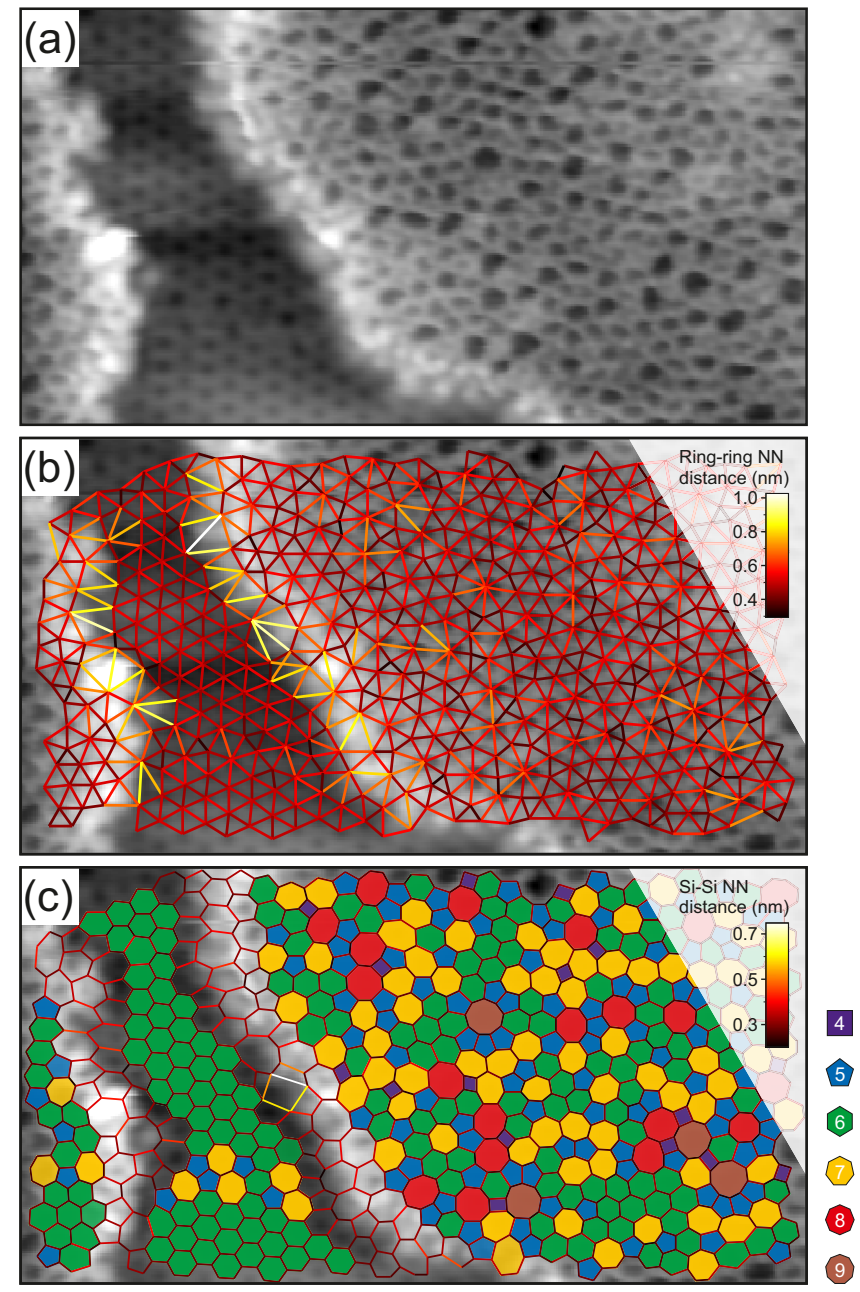

FIG. 1. (a) Ring resolved STM image of a ML-BL silica film transition region $\left(V_{S}=1 \mathrm{~V}, I_{T}=10 \mathrm{pA}, T=4.2 \mathrm{~K}\right.$, scan area $=$ $15.3 \mathrm{~nm} \times 8.1 \mathrm{~nm}$; applied filters: align rows, equalize). The STM image is superimposed by the color-coded ring-ring (b) and $\mathrm{Si}-\mathrm{Si}$ ring network model (c). The color codes for distances and ring sizes are provided at the right.

Here, we could resolve the vitreous silica bilayer film across such a single $\mathrm{Ru}(0001)$ step edge. In Fig. 2(a), the characteristic ring structure of the film is clearly resolved on both terraces. To resolve a step edge region is challenging, because of tip convolution effects and changes in the tip sample distance. To compensate for the height difference, we locally enhanced the image contrast for the network detection process. The filtered STM image is shown in Sec. 1 of the Supplemental Material. In Fig. 2(b), the STM image is overlaid with the ring-ring network model. In this case the ring-ring distances are uniformly distributed across the step edge region. Figure 2(c) shows the continuous nonperiodic network with four- to nine-membered rings. The network agrees very well with the proposed random network by Zachariasen and previous STM measurements on a single terrace $[29,43]$. The assigned rings form a continuous random network structure across the step edge. These observations confirm that within the scanned region, the film is intact across the $\mathrm{Ru}(0001)$ step edge. 

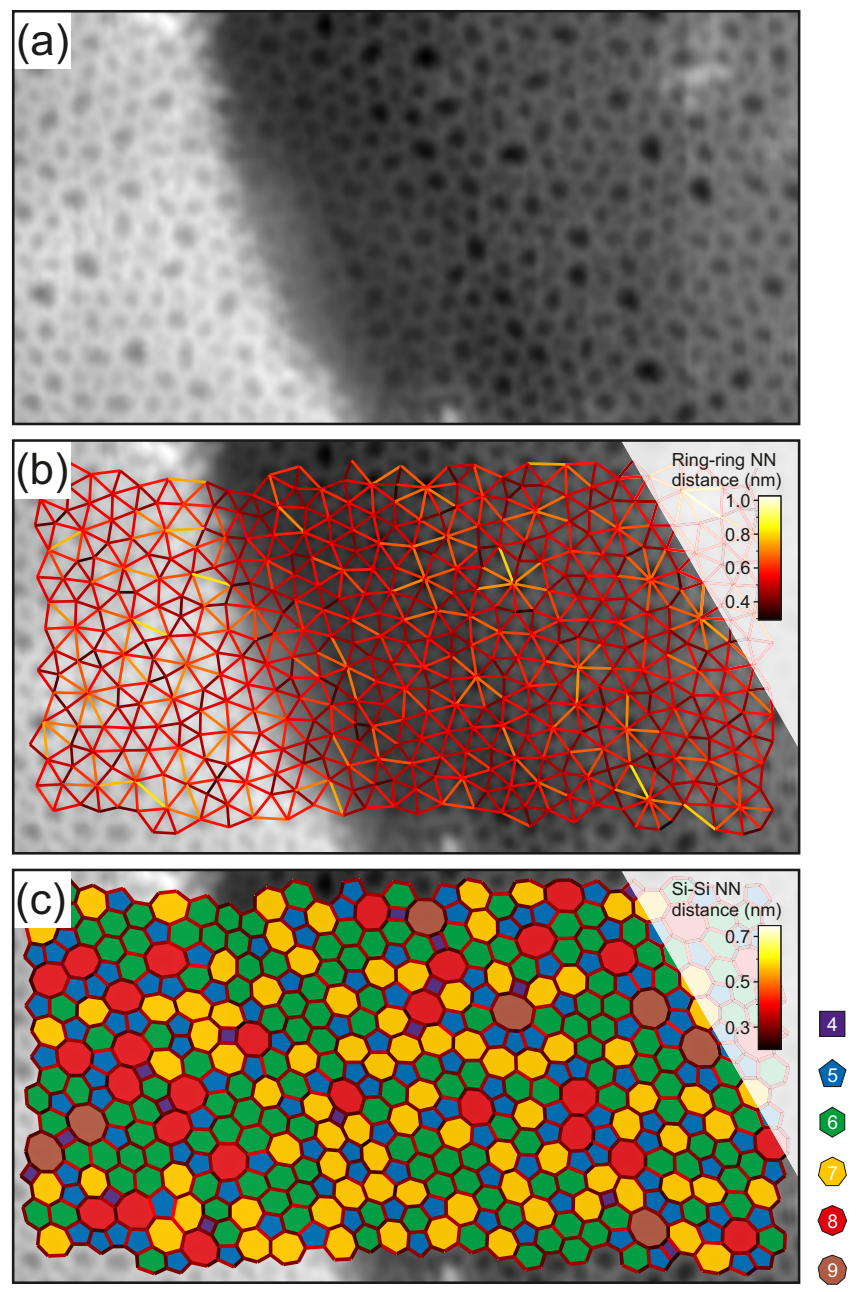

FIG. 2. (a) Ring resolved STM image of the silica film across a single $\mathrm{Ru}(0001)$ step edge $\left(V_{S}=2 \mathrm{~V}, I_{T}=400 \mathrm{pA}, T=295 \mathrm{~K}\right.$, scan area $=15.3 \mathrm{~nm} \times 8.1 \mathrm{~nm}$ ). (b), (c) The STM image, superimposed by the ring networks in analogy to Fig. 1 .

We use the detected network as a starting point for quantitative measurements of the structural features across the step. A detailed discussion is provided in Secs. 2-5 of the Supplemental Material. Both the average ring-ring distances and the average $\mathrm{Si}-\mathrm{Si}$ distances are in good agreement with literature values $[28,44-46]$. In the vicinity of the step, on the upper terrace slightly larger distances and on the lower terrace slightly shorter distances are detected. Yet, the quantitative analysis of the STM morphology reveals that the structural values of the silica bilayer across the step are in good agreement with literature values for flat vitreous silica films. This finding affirms a lateral smooth transition of the silica film from the upper to the lower terrace of the Ru substrate.

To study the nature of the silica network across the step, we performed DFT calculations of a crystalline bilayer system. Vitreous and crystalline network structures exhibit the same tetrahedral building block. To reduce the complexity of simulating an amorphous structure, the crystalline silica bilayer has been considered. This is justified by considering that vitreous and crystalline silica films have similar bending rigidity [47] and phonon spectra [48], both interact with the (a)

(b)

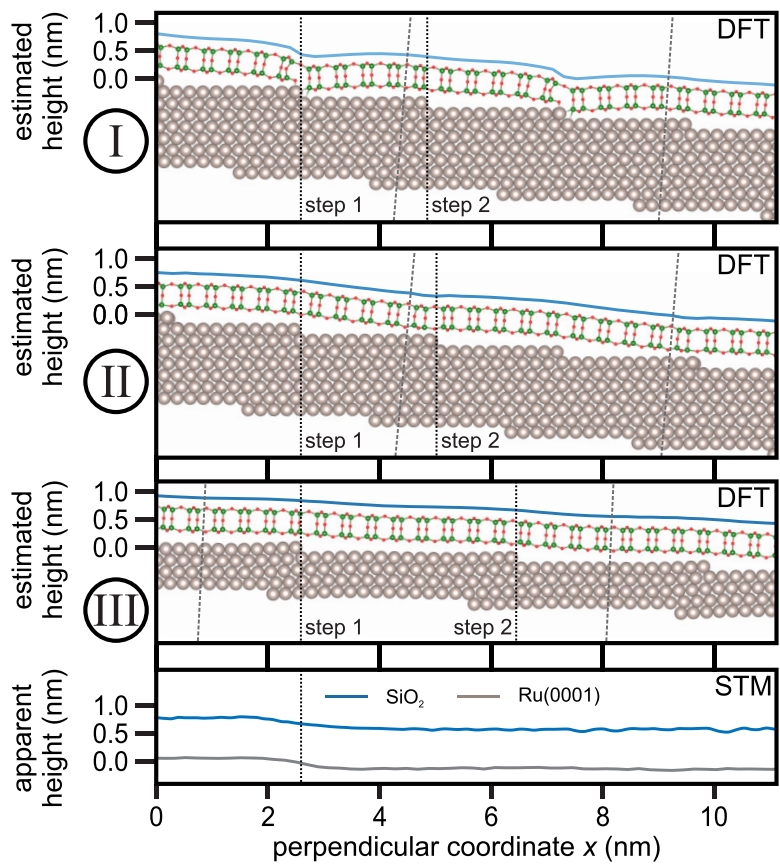

FIG. 3. DFT models of the crystalline silica bilayer across a $\mathrm{Ru}$ step edge. The topmost oxygen atom positions are plotted in comparison with STM line profiles. (a) Case I and (b) case II: DFT model on a Ru(101̄20) surface. (c) Case III: DFT model on a $\mathrm{Ru}(10 \overline{1} 31)$ surface. (d) STM line profiles of a Ru step edge covered with $\mathrm{SiO}_{2}\left(V_{S}=2 \mathrm{~V}, I_{T}=400 \mathrm{pA}, T=295 \mathrm{~K}\right)$. For comparison, the STM line profile of a bare $\mathrm{Ru}(0001)$ is plotted $\left(V_{S}=2 \mathrm{~V}, I_{T}=300\right.$ $\mathrm{pA}, T=295 \mathrm{~K})$. The vertical dotted lines mark the position of the substrate step edges. The inclined dotted lines mark the unit cell used for DFT calculations.

flat $\mathrm{Ru}$ surface via van der Waals forces, and the nature of the chemical bonds between a broken silica film and the $\mathrm{Ru}$ stepped surface is the same. DFT reveals the structure of both the upper and the lower layer of the bilayer system. In this study, we present three cases, which are shown in Fig. 3. The computational model for cases I and II consists of a seven-layer $(2 x 1)$ supercell $\mathrm{Ru}(10 \overline{1} 20)$ surface, exposing 1.9-nm-wide (0001) terraces separated by steps. In this model, a tensile strain of $2.7 \%$ in the direction parallel to the step and a compressive strain of $-1.3 \%$ perpendicular to the step is released on the $\mathrm{SiO}_{2}$ film. In reality, patched holes in the silica bilayer and various kinds of substrate step edges can cause strain in the film system. Case I and case II resulted from unconstrained relaxations, starting from different guesses: a broken structure in case I and a tilted undistorted bilayer in case II.

On top of the theoretical models in Fig. 3, we emphasized the topography of the silica bilayer by plotting the apical oxygen atom positions with an offset of $0.2 \mathrm{~nm}$. For comparison, line profiles from STM measurements are plotted for a bare $\mathrm{Ru}(0001)$ surface and the silica bilayer on $\mathrm{Ru}(0001)$ in Fig. 3(d). Details on the data extraction and zoomed sections of the line profiles are provided in Sec. 12 of the Supplemental Material. The apparent step height of the experimental profiles reflects the $\mathrm{Ru}(0001)$ interlayer spacing of $0.2 \mathrm{~nm}$.

In case I, Si-O bonds break at the step edge and bind to the substrate, as shown in Fig. 3(a). This pinning of the film to the 
substrate causes an almost steplike decrease of the line profile. The line profile exhibits a similar slope on the left of step 1 as the experimental derived curve in Fig. 3(d). The hump on the lower terrace, however, could not be observed experimentally. Due to the broken $\mathrm{SiO}_{2}$ bond in the lower layer of the silica bilayer we refer to the structure of case I as broken.

In case II, the bilayer covers the step edge in a carpetlike mode. The topography is smoother and less abrupt than for case I. In this aspect, the emphasized topography follows the step better than for case I. While at step 1, the topography of case II is in agreement with the STM line profile in Fig. 3(d), the trends of the theoretical and the experimental curve differ at step 2. Step 1 in case II could resemble the experimentally observed step edge. We refer to this structure of case II as bent. The bent structure is metastable with respect to the broken one by $7.9 \mathrm{meV} / \mathrm{SiO}_{2}$ unit.

We carefully checked the effect of the strain by applying both tensile and compressive strain (in the range 1\%-2\%) to the whole model. The tensile strain induces the $\mathrm{Si}-\mathrm{O}$ bond breaking and pinning to the $\mathrm{Ru}$ substrate, while compressive strain stabilizes the bent isomer. Alternative structural models of silica films with broken bonds at the step edge are intrinsically unstable or considerably higher in energy compared to the above presented models. Details on the structural features of cases I and II and broken films are provided in Secs. 7-9 of the Supplemental Material.

As mentioned above, for both cases I and II, the silica film does not mimic the underlying $\mathrm{Ru}$ step edges. The narrow $\mathrm{Ru}$ terraces can be the reason for this discrepancy. We therefore used the computational model of case III that consists of a four-layer $\mathrm{Ru}(10 \overline{1} 31)$ surface with 3.5-nm-wide terraces. On this surface, the lattice mismatch between silica and substrate in the direction perpendicular to the step is very small with $-0.2 \%$ and the interface is virtually strain-free.

The bent silica film geometry of case III is shown in Fig. 3(c). Interestingly, only the bent isomer is stable on the surface of case III, and any attempt to relax a pinned structure led to the spontaneous recombination of the broken $\mathrm{Si}-\mathrm{O}$ bonds. Since wider terraces better resemble the experimental conditions, this observation strongly supports the presence of the bent structure in experiment. While the bilayer seems quite rigid at step 1, it bends more at step 2 and its qualitative trend agrees with the experimental curve. In addition to the agreement of the emphasized topographies, the evolution of characteristic distances shows qualitatively a similar trend in the vicinity of step 2 . The ring-ring and $\mathrm{Si}$-Si distances increase on the upper and decrease on the lower terrace. A detailed analysis is provided in Sec. 15 of the Supplemental Material.

For all cases, the upper layer of the bilayer is intact, which supports the results from the lateral characterization by STM. While a continuous transition from the ML to the BL phase could not be detected, we gave evidence for the continuous coverage of a silica bilayer across a Ru step. The experimental findings are qualitatively in agreement with the modeled bilayer across step 2 in case III.

In comparison to graphene, the silica bilayer mimics the $\mathrm{Ru}$ step less accurately, as shown in Sec. 12 of the Supplemental Material. The observation is in line with the higher bending rigidity of silica compared to graphene [47,49]. This relation is an example of how the atomistic model is connected to macroscopic properties [11]. In analogy to studies of the macroscopic bending of thin plates more than 100 years ago [50,51], we provide an atomistic model for a freestanding silica bilayer that is exposed to bending stress in Sec. 6 of the Supplemental Material. For the supported silica bilayer, the substrate condition (e.g., the terrace width) and the mesoscopic structure might additionally affect the bilayer topography. As mentioned above, patched holes and substrate steps are possible influences. A brief excursus on this topic is provided in Sec. 14 of the Supplemental Material. Interesting future experiments include the observation of the silica bilayer on vicinal $\mathrm{Ru}$ surfaces [52,53] and parameter studies of different film preparation conditions.

The continuous coverage of the silica bilayer across $\mathrm{Ru}$ step edges can in principle have two origins. On the one hand, the film can cover the substrate continuously during deposition. For graphene such a unidirectional carpetlike mode is reported from upper to lower terraces [54]. On the other hand, the $\mathrm{Ru}$ step edges can diffuse underneath the silica bilayer due to oxygen coverage and annealing [55]. During graphene growth on $\mathrm{Ru}(0001)$ the $\mathrm{Ru}$ substrate and the Ru step geometry changes at the elevated temperature of $665^{\circ} \mathrm{C}$ [26]. The silica film in this study was annealed at more elevated temperatures in an oxygen atmosphere. Thus, the diffusion of $\mathrm{Ru}$ underneath the silica bilayer is a possible scenario, which can then result in continuous silica films across substrate step edges. To answer the question of the origin of the observed continuous silica film is an interesting scope for future experiments.

To summarize, atomic level STM investigations and DFT modeling of carpetlike and pinning modes have shown a continuous coverage of a vitreous bilayer silica film across a $\mathrm{Ru}$ substrate step edge. While a pinning of the lower layer to the substrate, not accessible to STM, cannot be excluded, the continuous random network ring structure when crossing the step edge proves the intact network structure of the upper layer of the vitreous silica bilayer.

This project has received funding from the European Research Council (ERC) under the European Unions Horizon 2020 Research and Innovation Program (Grant Agreement No. 669179). L.G. acknowledges support by the IMPRS for Elementary Processes in Physical Chemistry. S.T. and G.P. acknowledge the CINECA Centre for granting access to supercomputing resources through the ISCRA program.
[1] C. J. Heard, J. Čejka, M. Opanasenko, P. Nachtigall, G. Centi, and S. Perathoner, Adv. Mater. 31, 1801712 (2019).
[2] M. G. Blamire, J. L. MacManus-Driscoll, N. D. Mathur, and Z. H. Barber, Adv. Mater. 21, 3827 (2009). 
[3] C. Büchner, Z.-J. Wang, K. M. Burson, M.-G. Willinger, M. Heyde, R. Schlögl, and H.-J. Freund, ACS Nano 10, 7982 (2016).

[4] H.-J. Freund, M. Heyde, and C. Büchner, U.S. Patent No. 10,763,102 (2020).

[5] C. Büchner and M. Heyde, Prog. Surf. Sci. 92, 341 (2017).

[6] K. S. Novoselov, A. Mishchenko, A. Carvalho, and A. H. Castro Neto, Science 353, aac9439 (2016).

[7] B. V. Lotsch, Annu. Rev. Mater. Res. 45, 85 (2015).

[8] J. W. Reiner, F. J. Walker, and C. H. Ahn, Science 323, 1018 (2009).

[9] D. Kuhness, H. J. Yang, H. W. Klemm, M. Prieto, G. Peschel, A. Fuhrich, D. Menzel, T. Schmidt, X. Yu, S. Shaikhutdinov, A. Lewandowski, M. Heyde, A. Kelemen, R. Włodarczyk, D. Usvyat, M. Schütz, J. Sauer, and H.-J. Freund, J. Am. Chem. Soc. 140, 6164 (2018).

[10] A. L. Lewandowski, P. Schlexer, S. Tosoni, L. Gura, P. Marschalik, C. Büchner, H. Burrall, K. M. Burson, W.-D. Schneider, G. Pacchioni, and M. Heyde, J. Phys. Chem. C 123, 7889 (2019).

[11] R. Bennewitz, V. Barwich, M. Bammerlin, C. Loppacher, M. Guggisberg, A. Baratoff, E. Meyer, and H.-J. Güntherodt, Surf. Sci. 438, 289 (1999).

[12] C.-Y. Chang, H.-D. Li, S.-F. Tsay, S.-H. Chang, and D.-S. Lin, J. Phys. Chem. C 116, 11526 (2012).

[13] C. Tegenkamp, H. Pfnür, W. Ernst, U. Malaske, J. Wollschläger, D. Peterka, K. M. Schröder, V. Zielasek, and M. Henzler, J. Phys.: Condens. Matter 11, 9943 (1999).

[14] C. Schwennicke, J. Schimmelpfennig, and H. Pfnür, Surf. Sci. 293, 57 (1993).

[15] W. Hebenstreit, J. Redinger, Z. Horozova, M. Schmid, R. Podloucky, and P. Varga, Surf. Sci. 424, L321 (1999).

[16] F. Matthaei, S. Heidorn, K. Boom, C. Bertram, A. Safiei, J. Henzl, and K. Morgenstern, J. Phys.: Condens. Matter 24, 354006 (2012).

[17] M. E. Cañas Ventura, W. Xiao, P. Ruffieux, R. Rieger, K. Müllen, H. Brune, and R. Fasel, Surf. Sci. 603, 2294 (2009).

[18] F. Rossel, M. Pivetta, F. Patthey, E. Ćavar, A. P. Seitsonen, and W.-D. Schneider, Phys. Rev. B 84, 075426 (2011).

[19] I. Sebastian, T. Bertrams, K. Meinel, and H. Neddermeyer, Faraday Discuss. 114, 129 (1999).

[20] K. Meinel, A. Eichler, K.-M. Schindler, and H. Neddermeyer, Surf. Sci. 562, 204 (2004).

[21] E. Starodub, S. Maier, I. Stass, N. C. Bartelt, P. J. Feibelman, M. Salmeron, and K. F. McCarty, Phys. Rev. B 80, 235422 (2009).

[22] S. Saadi, F. Abild-Pedersen, S. Helveg, J. Sehested, B. Hinnemann, C. C. Appel, and J. K. Nørskov, J. Phys. Chem. C 114, 11221 (2010).

[23] C.-M. Seah, S.-P. Chai, and A. R. Mohamed, Carbon 70, 1 (2014).

[24] S. Marchini, S. Günther, and J. Wintterlin, Phys. Rev. B 76, 075429 (2007).

[25] J. Coraux, A. T. N'Diaye, C. Busse, and T. Michely, Nano Lett. 8, 565 (2008).

[26] S. Günther, S. Dähardt, B. Wang, M.-L. Bocquet, S. Schmitt, and J. Wintterlin, Nano Lett. 11, 1895 (2011).

[27] I. Palacio, A. Celis, M. N. Nair, A. Gloter, A. Zobelli, M. Sicot, D. Malterre, M. S. Nevius, W. A. de Heer, C. Berger, E. H.
Conrad, A. Taleb-Ibrahimi, and A. Tejeda, Nano Lett. 15, 182 (2015).

[28] L. Lichtenstein, M. Heyde, and H.-J. Freund, Phys. Rev. Lett. 109, 106101 (2012).

[29] L. Lichtenstein, C. Büchner, B. Yang, S. Shaikhutdinov, M. Heyde, M. Sierka, R. Włodarczyk, J. Sauer, and H.-J. Freund, Angew. Chem., Int. Ed. 51, 404 (2012).

[30] M. J. Prieto, H. W. Klemm, F. Xiong, D. M. Gottlob, D. Menzel, T. Schmidt, and H.-J. Freund, Angew. Chem., Int. Ed. 57, 8749 (2018).

[31] M. Heyde, M. Kulawik, H.-P. Rust, and H.-J. Freund, Rev. Sci. Instrum. 75, 2446 (2004).

[32] N. Nilius, A. Cörper, G. Bozdech, N. Ernst, and H.-J. Freund, Prog. Surf. Sci. 67, 99 (2001).

[33] G. Kresse and J. Furthmüller, Comput. Mater. Sci. 6, 15 (1996).

[34] G. Kresse and J. Furthmüller, Phys. Rev. B 54, 11169 (1996).

[35] M. Hacene, A. Anciaux-Sedrakian, X. Rozanska, D. Klahr, T. Guignon, and P. Fleurat-Lessard, J. Comput. Chem. 33, 2581 (2012).

[36] S. Maintz, B. Eck, and R. Dronskowski, Comput. Phys. Commun. 182, 1421 (2011).

[37] P. E. Blöchl, Phys. Rev. B 50, 17953 (1994).

[38] G. Kresse and D. Joubert, Phys. Rev. B 59, 1758 (1999).

[39] J. P. Perdew, K. Burke, and M. Ernzerhof, Phys. Rev. Lett. 77, 3865 (1996).

[40] S. Grimme, J. Comput. Chem. 27, 1787 (2006).

[41] S. Tosoni and J. Sauer, Phys. Chem. Chem. Phys. 12, 14330 (2010).

[42] See Supplemental Material at http://link.aps.org/supplemental/ 10.1103/PhysRevMaterials.5.L071001 for the detailed analysis of ring-ring and atomic distances.

[43] W. H. Zachariasen, J. Am. Chem. Soc. 54, 3841 (1932).

[44] K. M. Burson, L. Gura, B. Kell, C. Büchner, A. L. Lewandowski, M. Heyde, and H.-J. Freund, Appl. Phys. Lett. 108, 201602 (2016).

[45] R. L. Mozzi and B. E. Warren, J. Appl. Cryst. 2, 164 (1969).

[46] A. C. Wright, J. Non-Cryst. Solids 179, 84 (1994).

[47] C. Büchner, S. D. Eder, T. Nesse, D. Kuhness, P. Schlexer, G. Pacchioni, J. R. Manson, M. Heyde, B. Holst, and H.-J. Freund, Phys. Rev. Lett. 120, 226101 (2018).

[48] N. F. Richter, F. E. Feiten, J. Pal, A. Plucienik, E. Emmez, S. Shaikhutdinov, H. Kuhlenbeck, T. Risse, H.-J. Freund, I. Goikoetxea, R. Włodarczyk, and J. Sauer, J. Phys. Chem. C 123, 7110 (2019).

[49] A. Al Taleb, H. K. Yu, G. Anemone, D. Farías, and A. M. Wodtke, Carbon 95, 731 (2015).

[50] A. Föppl, Die wichtigsten Lehren der höheren Elastizitätstheorie (BG Teubner, 1907), Vol. 5.

[51] L. D. Landau and E. Lifshitz, Theory of Elasticity (Pergamon, New York, 1959).

[52] W. Hoffmann and C. Benndorf, Eur. Conf. Surf. Sci. 377-379, 681 (1997).

[53] W. Hoffmann and C. Benndorf, J. Vac. Sci. Technol. A 18, 1520 (2000).

[54] P. W. Sutter, J.-I. Flege, and E. A. Sutter, Nat. Mater. 7, 406 (2008).

[55] G. Held, S. Uremović, and D. Menzel, Surf. Sci. 331-333, 1122 (1995). 